\title{
Noninvasive Molecular Imaging Using Reporter Genes
}

\author{
Peter Brader ${ }^{1,2}$, Inna Serganova ${ }^{3}$, and Ronald G. Blasberg ${ }^{2-4}$ \\ ${ }^{1}$ Department of Radiology, Division of Molecular Imaging, Medical University of Vienna, Vienna, Austria; ${ }^{2}$ Department of Radiology, \\ Memorial Hospital, Memorial Sloan-Kettering Cancer Center, New York, New York; ${ }^{3}$ Department of Neurology, Memorial Hospital, \\ Memorial Sloan-Kettering Cancer Center, New York, New York; and ${ }^{4}$ Molecular Pharmacology and Chemistry Program, \\ Sloan-Kettering Institute, Memorial Sloan-Kettering Cancer Center, New York, New York
}

\begin{abstract}
Noninvasive reporter gene imaging is a component of molecular imaging. Reporter imaging can provide noninvasive assessments of endogenous biologic processes in living subjects and can be performed using different imaging modalities. This review will focus on radionuclide-based reporter gene imaging as developed and applied in preclinical and clinical studies. Examples of different reporter systems are presented, with a focus on human reporter systems. Selected applications are discussed, including adoptive cell therapies, gene and oncoviral therapies, oncogenesis, signal pathway monitoring, and imaging drug treatment. Molecular imaging, and noninvasive reporter gene imaging in particular, are making important contributions to our understanding of disease development, progression, and treatment in our current era of molecular medicine and individualized patient care.
\end{abstract}

Key Words: molecular imaging; oncology; PET; gene therapy; reporter gene imaging

J Nucl Med 2013; 54:167-172

DOI: 10.2967/jnumed.111.099788

The convergence of imaging technology and the disciplines of molecular and cell biology in the mid-1990s provided the foundation for the development of noninvasive molecular imaging using reporter genes. This development was originally stimulated by programs funded by the National Institutes of Health and the Department of Energy. This initiative has now spread globally throughout Europe and Asia, and new molecular imaging societies and new journals have been developed to address this rapidly expanding discipline.

Reporter gene imaging is only a single component of molecular imaging. Researchers developed reporter genes in the 1980s, using the bacterial $\beta$-galactosidase (1) and chloramphenicol acetyltransferase (2) genes, to study various cellular processes both in vitro and in vivo. However, visualization of these reporter enzymes required postmortem tissue sampling and processing $(1,2)$. Advances in cell biology eventually stimulated the development of novel noninvasive visualization systems that could provide accurate and sensitive measurements in animal models of human disease.

Noninvasive molecular imaging in living animals is the direct result of significant developments in several imaging technologies,

\footnotetext{
Received Jul. 31, 2012; revision accepted Nov. 26, 2012.

For correspondence or reprints contact: Ronald G. Blasberg, Departments of Neurology and Radiology, MH (Box 52), Molecular Pharmacology and Chemistry Program, SKI, Memorial Sloan-Kettering Cancer Center (MSKCC), 1275 York Ave., New York, NY 10021.

E-mail: blasberg@neuro1.mskcc.org

Published online Jan. 14, 2013.

COPYRIGHT (C 2013 by the Society of Nuclear Medicine and Molecular Imaging, Inc.
}

including MR imaging (3), radionuclide imaging (quantitative autoradiography, $\gamma$-camera imaging, and PET) (4), and optical imaging of small animals (5). These developments occurred more or less in parallel to each other and were largely independent of the advances that were occurring in genetics and in molecular and cell biology during the 1980s and early 1990s. Each of these imaging technologies also had important antecedents. For example, the development of radionuclide-based molecular imaging is founded on the radiotracer principle, which was first described by George de Hevesy. In 1935 he published a letter in Nature on the tracer principle using ${ }^{32} \mathrm{P}(6)$, and he was awarded the Nobel Prize in chemistry in 1943. The tracer technique was later adapted for many applications in physiology and biochemistry, as well as in functional diagnosis and in nuclear medicine and molecular imaging.

\section{REPORTER GENE IMAGING PARADIGMS}

A common feature of all reporter constructs is that the complementary DNA expression cassette, containing the reporter transgenes of interest (e.g., herpes simplex virus 1 thymidine kinase [HSVl-tk]), can be placed under the control of specific promoter-enhancer control elements. The versatility of reporter constructs is due in part to their modular design, since arrangements in the expression cassette can be varied to some extent. For example, reporter genes can be constructed to be "always on" using constitutive promoters (such as LTR, RSV, CMV, PGK, and EF1). Alternatively, the promoter-enhancer elements can be constructed to be inducible and sensitive to activation and regulation by specific endogenous transcription factors and promoters (factors that bind to and activate specific enhancer elements in the regulatory region of the reporter construct, leading to the initiation of reporter gene transcription). These strategies have been widely applied in both optical imaging $(7,8)$ and radionuclide-based imaging (9) and to a lesser degree in MR imaging (3).

\section{MODALITIES}

\section{In Vivo Optical Imaging}

Optical reporter proteins can emit light in the visible range (300-600 nm), either through interaction with specific substrates (bioluminescence) or after excitation with light of a specific wavelength (fluorescence). The emitted light is captured by a sensitive chargecoupled-device camera (10). Both bioluminescence and fluorescence reporter genes have advantages and disadvantages. Bioluminescence imaging is the most affordable and cost-effective real-time analysis of gene expression in small animals; bioluminescence imaging has excellent sensitivity and is user-friendly. Until recently, tomographic images and volumetric semiquantification was limited because of light scatter, differential light absorption, and lack of depth information. 
Fluorescence reporter genes were used extensively in the past to image molecular events in single live cells but are less adaptable to imaging live animals - although issues related to autofluorescence are being addressed using spectral unmixing techniques (11). Bioluminescence imaging is more sensitive for small-animal imaging than is fluorescence imaging, because of high signal-to-background ratios and absence of autoluminescence (12). Although optical imaging provides outstanding sensitivity or resolution, it is limited in translation to clinical applications (13).

\section{MR Imaging}

Compared with radionuclide or optical reporter genes, the development and application of MR reporters is more limited (3). Three classes of MR reporter genes, based on their interaction at the molecular level, have been described (14): enzyme-based cleavage of functional groups that block water (proton) exchange, protein binding, or MR imaging contrast agents; the expression of surface receptors that enable the binding of specific MR imaging contrast agents; and the expression of para- and antiferromagnetic proteins involved with iron metabolism, such as tyrosinase and ferritin. MR imaging is probably the least used reporter gene imaging technique for small animals; it has comparatively low sensitivity but higher resolution than radionuclide imaging. MR-based reporter gene imaging has not occupied center stage, and no clinical studies have been reported.

\section{Radionuclide Imaging}

The radionuclide imaging class of reporter genes encodes for proteins that interact with and trap radiolabeled molecules, resulting in localized accumulation of radioactivity that reflects the expression level of the reporter gene. One of the first noninvasive reporter gene imaging paradigms was based on HSVl-tk and was described in 1995 (15). The HSVl-tk reporter system is a radiotracer enzymatic assay; it is similar to the FDG-hexokinase paradigm that is widely used to image glucose utilization. Other reporter strategies use a transporter or a receptor to selectively trap a radiolabeled probe, such that the level of accumulated radioactivity reflects the expression of the reporter gene. All these reporter systems involve the coupling of a reporter gene with a specific reporter substrate - a complementary pair. Radionuclide-based reporters have the potential for immediate clinical application and are the focus of current development.

\section{HSV1-tk and Other Enzyme-Based Genes}

The initial and most widely used radionuclide-based reporter system is $H S V 1$ - $t k$ (15) coupled to ${ }^{124} \mathrm{I} /{ }^{18} \mathrm{~F}-2$ '-fluoro-2'-deoxy-1$\beta$-D-arabinofuranosyl-5-iodouracil (FIAU) or ${ }^{18} \mathrm{~F}-9$-[4-fluoro-3(hydroxymethyl)butyl]guanine (FHBG). Unlike mammalian $T K$, the HSVI-tk and HSVI-tk $k_{s r 39}$ enzymes are much less substratespecific; they phosphorylate modified thymidine analogs (e.g., FIAU and FHBG), as well as antiviral drugs (acyclovir, ganciclovir, and penciclovir). HSV1-tk had been used as a suicide therapeutic gene well before its use as a reporter gene. The Varicella zoster virus thymidine kinase gene and bicyclic nucleoside probes represent a relatively new and not fully validated PET reporter system (16).

\section{Human Reporter Gene Systems}

Recently, enzyme-based human reporter genes have been developed and validated to avoid potential immunologic reactions to HSV1-tk protein. They include human mitochondrial thymidine kinase-2 (hmtk2) (17) and several mutants (18), as well as a mutant of human deoxycytidine kinase $(h d C K)$ using clinically available pyrimidine-based radiotracers (19). Other reporter systems have also been described: NIS (rat sodium iodide symporter (20)), hNET (human norepinephrine transporter (21)), hSSTR2 (human somatostatin receptors (22)), hCEA (human carcinoembryonic antigen [CEA] (23)), and $D 2 R$ (rat dopamine $\mathrm{D}_{2}$ receptor (24)).

$h m t k 2 / h \Delta T K 2$. A human $\Delta$-mitochondrial thymidine kinase type 2 ( $h \Delta T K 2$ ) was developed and can be imaged with pyrimidine-based radiotracers. However, cells expressing $h \Delta T K 2$ show relatively low levels of pyrimidine radiotracer accumulation compared with HSVI-tk (one sixth to one tenth) (17). Further enzyme engineering resulted in the development of a mutant $h T K 2-N 93 D /$ $L 109 F$ with the ability to phosphorylate ${ }^{18} \mathrm{~F}-\mathrm{FMAU}$ (18).

$h d C K$. $h d C K$ was developed as a PET reporter gene. $h d C K$ is capable of converting deoxycytidine, deoxyadenosine, deoxyguanosine, and several pyrimidine-based cytotoxic drugs (including cytosine-arabinoside and 2 '-difluorocytosine) into their monophosphate forms. $h d C K$ along with ${ }^{18} \mathrm{~F}-2^{\prime}$-deoxy-2'-fluoroarabinofuranosylcytosine was developed as a new reporter system, and $h d C K$ mutants were shown to phosphorylate pyrimidine-based radiotracers at levels comparable to that of wild-type HSVI-tk (19). The in vitro radiotracer accumulation and in vivo imaging studies demonstrated the advantage of this mutant reporter gene over its native predecessor, because of a thymidine-permissive conformation of this enzyme that allows phosphorylation of FIAU and FEAU.

$h N I S$. $h N I S$ is an intrinsic transmembrane glycoprotein and member of the sodium/solute symporter family (25). hNIS is naturally expressed in the thyroid gland and stomach and, to a lesser extent, in salivary and lactating mammary glands, the choroid plexus, kidney epithelial cells, and the placenta (20). The $h N I S$ has many advantages as a reporter gene, primarily because of the wide availability of substrates already approved for clinical use (both for $\gamma$-camera $\left[{ }^{123} \mathrm{I}^{-},{ }^{131} \mathrm{I}^{-}\right.$, and $\left.{ }^{99} \mathrm{~m} \mathrm{TcO}_{4}{ }^{-}\right]$and PET $\left[{ }^{124} \mathrm{I}^{-}\right.$

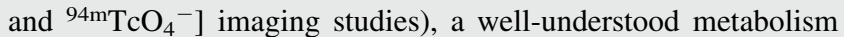
and clearance of its substrates from the body, and the fact that radiochemistry laboratory facilities do not need to be nearby. In addition, all nuclear medicine departments have access to a $\gamma$ camera or a SPECT system (and many have PET systems). Nevertheless, hNIS also has limitations; high background activity in the thyroid, stomach, intestine, and other tissues can obscure reporter imaging (limit the sensitivity and specificity) in or adjacent to these tissues, and iodide and pertechnetate rapidly clear from $h N I S$-expressing cells and tissues as medium and blood levels fall.

hNET. hNET is a transmembrane protein, is one of several monoamine transporters involved in the transport of norepinephrine, epinephrine, and dopamine (26), and functions as a rapid reuptake system located at or near presynaptic terminals. Several radiolabeled probes are used clinically $\left({ }^{123} \mathrm{I},{ }^{131} \mathrm{I},{ }^{123 / 124} \mathrm{I}\right.$-metaiodobenzylguanidine, and ${ }^{11} \mathrm{C}$-ephedrine), and the small size of the $h N E T$ reporter gene cassette allows it to be easily incorporated into the delivery vehicle (27). The successful imaging of $h N E T$ expression in neuroendocrine tumors and altered sympathetic enervation of the heart have led to the suggested application of $h N E T$ as a human reporter gene $(21,28)$. Sequential imaging of animals bearing both $h N E T$ reporter-transduced and wild-type xenografts clearly showed the advantage of late imaging (27). The background-corrected radioactivity profile of the $h N E T$-transduced xenograft shows a peak value at $8 \mathrm{~h}$ and a slow exponential washout (half-life, $63 \mathrm{~h}$ ), compared with the more rapid washout from the nontransduced xenograft (half-life, $12 \mathrm{~h}$ ). Calculating the fraction of measured radioactivity that represents trapped (nonbackground) radioactivity shows that measured values can 
be misleading, particularly at early times (at 1,2 , and $8 \mathrm{~h}$, the trapped radioactivity is $\sim 14 \%, 52 \%$, and $83 \%$ of the measured radioactivity, respectively). The radioactivity ratio, comparing transduced-to-nontransduced xenografts, is also highly time-dependent (increasing from 1.2:1 at $1 \mathrm{~h}$ to $6.5: 1,14: 1$, and 28:1 at 8, 24 and $48 \mathrm{~h}$, respectively, after the injection of ${ }^{124}$ I-metaiodobenzylguanidine). These results highlight the importance of the imaging time when single-time experiments are compared (27).

hSSTR2. The somatostatin receptors are G-protein-linked transmembrane pass receptors. The expression of 1 of the 6 SSTr genes, subtype 2, is largely restricted to the pituitary, although other tissues express low levels of this protein (29). Although the hSSTr2 gene was originally described as a promising reporter gene in 1999 (29) and again in 2005 (30), image quality remained suboptimal using ${ }^{111} \mathrm{In}$ diethylenetriaminepentaacetic acid-octreotide, ${ }^{99 \mathrm{~m}} \mathrm{Tc}-\mathrm{P} 829,{ }^{188} \mathrm{Re}-$ P829, ${ }^{99 m}$ Tc-P2045, or ${ }^{94 m}$ Tc-demotate-1 radioligands. This situation has now changed significantly with the availability of ${ }^{68} \mathrm{Ga}$-DOTATOC, ${ }^{68} \mathrm{Ga}$-DOTATATE, and ${ }^{111}$ In-DOTABASS, due to high specific uptake and comparatively rapid body clearance and low background activity. These peptide-chelate conjugates were originally designed for radiotherapy of SSTr2-expressing tumors. DOTATOC and DOTATATE have been classified as receptor agonists, whereas DOTABASS is an antagonist. They have undergone extensive clinical testing in both Europe (31) and the United States (32) and are being developed as clinical imaging and targeted radiotherapeutic agents.

Because ligand binding to the hSSTr2 expressed on transduced cells could potentially perturb the biology of these cells through Gprotein-coupled receptor signaling (as was shown for the $D 2 R$ $(24,33)$ ), this issue must be addressed. In vitro, activation of SSTr2 on tumor cells results in cell growth arrest, whereas activation of SSTr 5 results in cell proliferation (34). We observed that SSTr2 transduction had variable effects on xenograft growth rates; these effects varied with cell type but not with the level of SSTr2 expression (35).

$h C E A$. A recombinant reporter gene based on the CEA was developed for imaging. The CEA-based reporter gene can be detected by molecular imaging using radiolabeled anti-CEA antibodies as a reporter probe. In vivo small-animal PET using a ${ }^{124}$ I-labeled anti-CEA scFv-Fc antibody visualized CEA-transfected Jurkat $\mathrm{T}$ cell xenografts (23).

$D 2 R . D_{2} R$ is also a potential reporter gene because of the availability of ${ }^{18} \mathrm{~F}$-fluoroethylspiperone, a well-studied probe for PET imaging of the dopaminergic system in humans. However, only the rat $D_{2} R$ reporter system has been studied, and potential problems such as occupancy of the receptor by endogenous natural ligands and adverse biologic effects on transduced cells have been raised. To address this concern, the group at the University of California at Los Angeles developed a signaling-inert mutant variant $\left(D 2 R_{80 A}\right)$ as a reporter gene and visualized gene expression with ${ }^{18} \mathrm{~F}$-fluoroethylspiperone (24). They showed that the $D 2 R_{80 A}$ variant has in vitro ${ }^{3} \mathrm{H}$-spiperone binding parameters similar to those of wild-type $D 2 R$, but there was complete uncoupling of ligand binding from activation of G-protein-linked signaling. Unfortunately, the nonhuman origin of the dopamine D2R80A receptors does not favor their use in clinical reporter gene imaging studies, and human $h D 2 R$ equivalents will have to be developed.

\section{Comparisons}

Every reporter gene has its advantages and disadvantages. HSVI-tk has a greater potential to elicit an immune reaction than human reporter systems. The expression of a transporter or a receptor on the surface of all cells involves a complex process of protein trafficking and membrane integration that may affect reporter readout and quantitation. An enzyme-based reporter would be expected to produce a higher signal than a receptor-based reporter because of enzymatic or catalytic trapping of the radiolabeled substrate, resulting in signal amplification. On the other hand, intracellular enzymebased reporters may be limited by transport of the radiolabeled substrate into transduced cells. For transporter and receptor-based reporter systems, the ligand-binding domain is located on the external plasma membrane (27) and does not require radioligand permeation of the cell membrane to bind to the receptor.

\section{SELECTED APPLICATIONS FOR CLINICAL TRANSLATION}

Molecular imaging paradigms involving reporter genes, although first applied to noninvasive in vivo imaging of small animals, are now being translated into clinical imaging paradigms. Although only 5 human reporter gene studies have been reported (36-40), translation is expected to expand and establish new standards of medical practice in the future. However, reporter gene imaging studies will always be more limited in patients than in animals, because of the need to transduce the target tissue or cells with specific reporter constructs or to produce transgenic animals bearing the reporter constructs.

\section{Gene and Oncoviral Therapy: Clinical Trials}

The promise of gene therapy and oncolytic viral therapy has yet to be realized, although both have been under investigation for decades. Monitoring the level of transgene expression or the distribution of oncolytic viruses would provide important, clinically relevant information that can be obtained only by noninvasive imaging. Preclinical animal studies have clearly shown that reporter imaging can track transgene expression and monitor oncolytic viruses (41). For example, $H S V 1$ - $t k$ can be used as a therapeutic gene as well as a reporter gene. Although HSV1-tk has been used extensively in gene therapy clinical trials performed in the United States and Europe (42,43), only 3 clinical HSVI-tk gene imaging studies (Fig. 1) (3638 ) and 1 hNIS gene imaging study (40) have been reported. Unfortunately, the pharmaceutical industry has been unwilling to image the magnitude of HSV1-tk expression in its clinical trials, even though the sensitivity of ${ }^{124}$ I-FIAU PET HSVI-tk expression has been shown to be more than 10-fold greater than that required for a therapeutic effect (Ronald G. Blasberg et al., unpublished data, April 1997).

\section{Adoptive Therapies: Clinical Trials}

A noninvasive method for repetitive evaluation of adoptively administered cells (e.g., bone marrow transplantation and immune cell-, stem cell-, and progenitor cell-based therapies) would benefit the assessment of current adoptive therapies, as well as future adoptive therapies using stem cells. The reporter systems that are being explored most intensively are adoptive therapy strategies to treat cancer (42) and heart disease (44). Individual patient monitoring would contribute to patient management by visualizing the trafficking, homing-targeting, and persistence of adoptively administered cells (using constitutive reporters) and would assess their functional and activation status (using inducible reporters, where the promoterenhancer elements are regulated by specific endogenous transcription factors). For example, our group has monitored and assessed T-cell receptor-dependent activation in vivo using noninvasive PET (45). Such studies would significantly aid in the clinical implementation and management of new therapeutic approaches based on the adoptive transfer of immune cells, progenitor cells, and stem cells. 


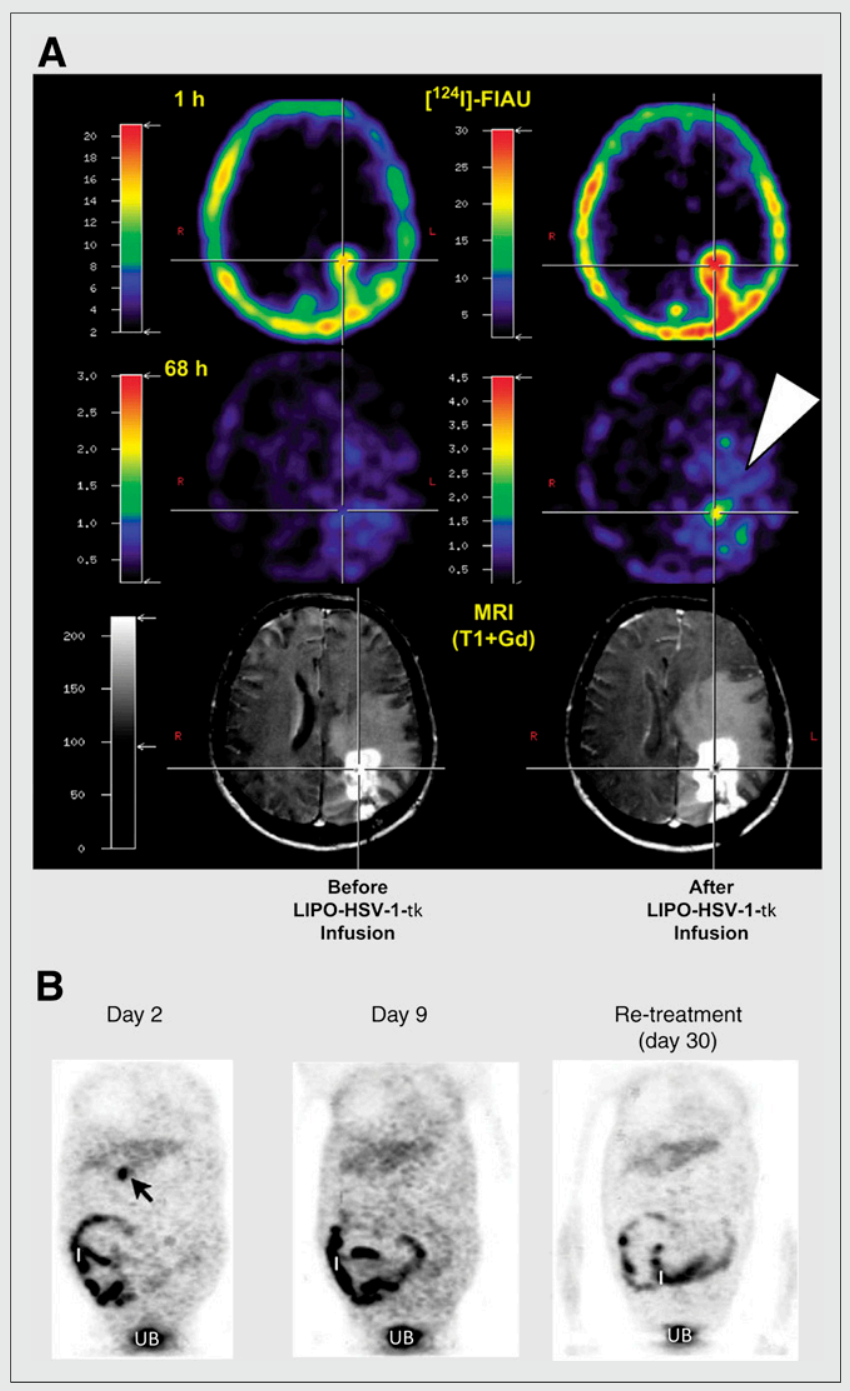

FIGURE 1. Clinical studies. (A) HSV1-tk reporter gene imaging in patients after liposome-HSV-1-tk complex transduction. Shown is coregistration of ${ }^{124}$ I-FIAU-PET and MR imaging before (left column) and after (right column) HSV1-tk vector injection. A region of specific ${ }^{124}$ I-FIAU retention (at $68 \mathrm{~h}$ ) within tumor is visualized (arrowhead). This tumor region showed signs of necrosis (cross hairs, right column) after ganciclovir treatment. However, only 1 of 5 patients showed ${ }^{124}$ I-FIAU-associated radioactivity localized at site of HSV1-tk vector injection. (Adapted from (36).) A similarly designed study, using ${ }^{123}$-FIAU and SPECT to monitor HSV1716 replication before and after administration of virus in 8 patients with high-grade gliomas, also failed to detect HSV1716-tk expression (37). (B) Adenoviral transgene (HSV1-tksr39) expression in patients with liver cancer. Coronal PET images $1.5 \mathrm{~h}$ after injection of ${ }^{18} \mathrm{~F}-\mathrm{FHBG}$ were obtained 2 and $9 \mathrm{~d}$ after first dose of AdCMVtk and $2 \mathrm{~d}$ after readministration (day 30 ) of adenoviral vector in same lesion. Specific ${ }^{18} \mathrm{~F}-\mathrm{FHBG}$ accumulation in treated tumor can be seen only at day 2 (arrow), and no specific accumulation can be seen either at day 9 or in retreatment. Radioactivity is observed in intestine (I) and urinary bladder (UB), as result of normal physiologic clearance of tracer. Late images obtained up to $6.5 \mathrm{~h}$ after injection did not show specific tracer accumulation in lesion either on day 9 or in retreatment study (images not shown). (Adapted from (38).)

Such imaging strategies have been widely applied in experimental animals, using both optical and radionuclide-based reporter imaging (8). Only a single clinical study has been reported, in which a patient with glioblastoma multiforme was infused with autologous cytolytic CD8 $+\mathrm{T}$ cells (CTLs) genetically engineered to express a tumortargeting receptor and the HSVI-tk reporter gene (39). After tumor resection approximately $1 \times 10^{9}$ CTLs were infused into the oper-

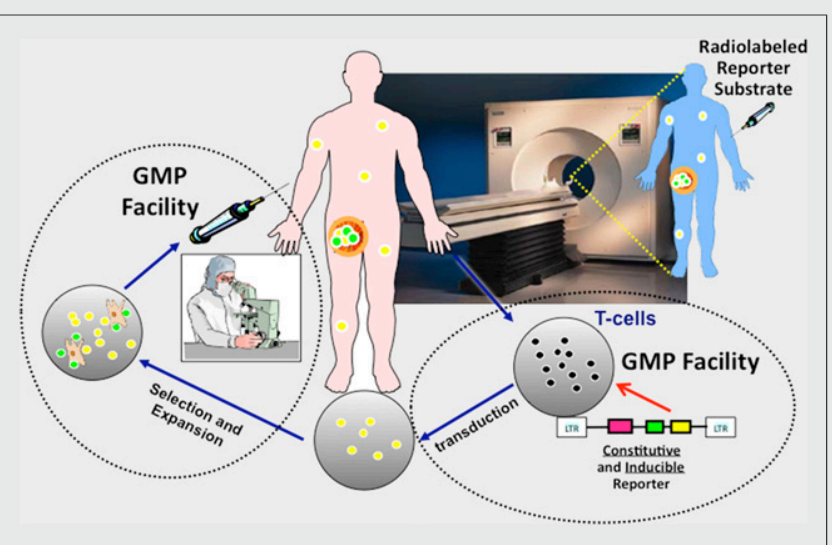

FIGURE 2. Infrastructure to image T-lymphocyte trafficking, targeting, and activation. Therapeutic antitumor immunity depends on highly migratory T-cell populations capable of trafficking between lymphoid and tumor tissue sites as well as infiltration and activation within microanatomic structure of tumors. Stable genetic labeling of adoptively transferred cells with various reporter genes can be used to circumvent temporal limitations of ex vivo radiolabeling. Peripheral human $T$ cells can be harvested from individual patients, transduced in GMP facility with vectors bearing both constitutive and activation-inducible reporter genes, and put through a round of selection and expansion. Then, these autologous genetically labeled tumor-specific $T$ lymphocytes can be reintroduced into same donor patient. After administration of radiolabeled reporter-specific substrates, adoptively administered $\mathrm{T}$ cells can be monitored with respect to their trafficking through body, their targeting and persistence in tumor, and their activation and functional status in tumor. T-cell trafficking and activation can be monitored in vivo noninvasively and sequentially with PET on daily or weekly basis, depending on half-life of radionuclide used to label reporter substrate. Thus, reporter gene imaging allows for reliable assessments of adoptive immunotherapy through repetitive visualization of cellular trafficking, persistence, proliferation, and function at target site.

ative site, and ${ }^{18}$ F-FHBG PET scans were able to detect the infused CTLs. Several other clinical trials are open, and others are being initiated. However, a limitation of such studies is the cost and required institutional infrastructure (Fig. 2).

\section{Imaging Oncogenesis and Signaling Pathways}

Genetic mouse models including reporter genes allow examination of the consequence of a specific gene alteration on the formation of tumors in their normal tissue environment and the onset and temporal dynamics of signal pathway changes in real time using noninvasive imaging. A proof-of-principle study that used bioluminescence imaging to detect and measure K-Ras-dependent lung tumor genesis involved genetically altered mice (46). This approach is highly versatile and easily translatable to other mouse models of cancer. However, reporter gene imaging of oncogenesis and tumor development can be performed only on animal models, usually with low-cost optical systems in mouse models.

Other examples of imaging oncogenesis include platelet-derived growth factor (PDGF)-induced gliomas (47), the PSA-luc prostate cancer model (48), and the pVEGF-TSTA-luc reporter for a mammary tumorigenesis model (49). Numerous reporter systems have been developed to image activation of specific signaling pathways, including endogenous regulation of transcription (an endogenous transcription factor drives an inducible reporter) $(45,50)$, posttranscriptional modulation of translation (51), and protein-protein interactions (52).

\section{Imaging Drug Treatment}

A strong rationale exists for assessing the effects of drugs that target or affect specific signaling pathways (e.g., PDGF/EGFR/HER2 signaling or Ras/Raf/MEK/Erk- and PKB/Akt/mTOR-mediated 
pathways) by noninvasive imaging. The development and use of reporter imaging (and direct imaging) paradigms to evaluate the efficacy of molecule-targeted therapies is being pursued by many groups. Specific drugs that are known (or thought) to target specific signaling pathways and downstream effectors can be assessed in reporter-expressing animal models or transgenic/oncogenic reporter animals. For example, the E2F-reporter mouse model was used in longitudinal preclinical studies to study the effects of treatment with a drug (PTK787/ZK222584) that inhibits the PDGF receptor (47). The E2F-reporter mouse model makes it possible to investigate the importance of PDGF-related signaling pathways in glioma maintenance and to image the effects of drug treatment. Other examples include the p53- and HSP90-sensitive reporter systems $(50,53)$.

\section{Translational Infrastructure: Good Manufacturing Practice (GMP) Facilities for Clinical Studies (Biologics and Radiopharmaceuticals)}

GMPs for clinical cell engineering and cell-based therapy of patients requires an institutional infrastructure that conforms with city, state, and federal regulations. The operational structure for cell-based GMP and clinical activities include the following: ex vivo expansion and transduction of a patient's cells; the production of ancillary cell lines and retroviral or lentiviral packaging cell clones with the creation of master cell banks; the production of viral stocks for subsequent cell transduction of cell-targeting, therapeutic, or reporter genes; and the production of clinical-grade plasmid DNA for vaccines and for generation of vector stocks. A schematic outline is shown in Figure 2. A quality control unit is essential to oversee several areas: first, the development, maintenance, and implementation of all standard operating procedures and batch records; second, the continual monitoring of the facility's environment; third, the review and release of the results of assays performed on critical raw materials (e.g., culture media and sera; manufactured products such as viral stocks, packaging cells, and master cell banks; and expanded and genetically engineered patient cells, plasmid DNA, and vaccines); fourth, maintenance of the calibration programs of all pieces of equipment; and fifth, maintenance of personnel training files. The batch records and manufacture records should be independently reviewed by the institutional office of clinical research. For the preparation of radiopharmaceuticals for clinical trials and for the routine practice of medicine, the institutional infrastructure must conform with both local and federal regulations. In the United States, this conformance is governed by a combination of U.S. Pharmacopeia (USP) chapters (notably, USP $<823>$ Radiopharmaceuticals for PET-Compounding and USP $<797>$ Pharmaceutical Compounding-Sterile Preparations), USP drug monographs, Food and Drug Administration GMP regulations (title 21 of Code of Federal Regulations part 212 for radiopharmaceuticals), and the manufacturing procedures and quality assurance controls that are claimed in investigational new drug applications. For clinical trials of investigational radiopharmaceuticals, strict compliance with USP $<823>$ is required. USP $<823>$ refers extensively to USP $<797>$, which considers radiopharmaceuticals as low-risk-level compounded sterile preparations that should be compounded in a certified class 5 primary engineering control located in a class 8 or better air environment. A quality control area adjacent to the radiochemistry GMP facility is necessary to provide the required pre- and postrelease quality testing for each manufactured drug. Batch records and manufacture records should be independently reviewed by the institutional office of clinical research. Two additional mechanisms that facilitate the initiation of clinical studies are radioactive drug research committees and explor- atory investigational new drug applications. Under title 21 of Code of Federal Regulations part 361.1, the Food and Drug Administration describes regulations that in effect delegate the oversight for radiopharmaceutical research to specially constituted institutional committees: radioactive drug research committees. Because the mechanism of these committees is not appropriate for first-in-humans studies, the exploratory investigational new drug has recently been developed as an alternative by the Food and Drug Administration. Under the exploratory investigational new drug, an investigator may combine early human studies at a phase 0 , or microdose, level as a basis for getting important information that may provide drug developers with an early indication of whether drugs (and radiopharmaceuticals) are promising (or lack promise) for further development.

\section{FUTURE}

Our understanding of disease processes will continue to expand rapidly as molecular-genetic studies continue to explore the basis of disease development and progression. The era of molecular medicine has begun, and the benefits to individual patient care are expected to be realized soon. Six complementary human reporter systems (gene + probe pairs) have been proposed as candidates for future reporter gene imaging in patients. Importantly, these human genes are less likely to be immunogenic than are the reporter genes currently used in animals (e.g., viral thymidine kinases, luciferases, and fluorescent proteins). Once a complementary reporter pair (gene + probe) has been approved for human studies, the major regulatory focus will shift to the particular backbone and regulatory sequence of the reporter construct and to the vector used to target reporter transduction to specific cells or tissue, both ex vivo and in vivo. Ideal vectors for targeting specific organs or tissues (tumors) do not currently exist, although this is an active area of human gene therapy research. Although 3 of the 4 clinical studies were performed on patients with brain tumors, a good brain radiotracer-based reporter system has yet to be developed and validated, primarily because of limited reporter substrate penetration of the intact blood-brain barrier.

The development and validation of versatile and sensitive noninvasive assays that do not require tissue samples will be of considerable value. Molecular imaging, and noninvasive reporter gene imaging in particular, will make important contributions to monitoring molecular-genetic and cellular processes both in animal models and in human subjects and will be used for monitoring and optimizing new treatment strategies.

\section{DISCLOSURE}

This work was supported by NIH research grants 2P50CA086438-16, 1R01CA163980-01A1, and 5P01CA09406012. Financial support was also provided by the Federal Ministry of Economy, Family, and Youth and the National Foundation for Research, Technology, and Development of Austria. No other potential conflict of interest relevant to this article was reported.

\section{REFERENCES}

1. Forss-Petter S, Danielson PE, Catsicas S, et al. Transgenic mice expressing betagalactosidase in mature neurons under neuron-specific enolase promoter control. Neuron. 1990;5:187-197.

2. Overbeek PA, Chepelinsky AB, Khillan JS, Piatigorsky J, Westphal H. Lens-specific expression and developmental regulation of the bacterial chloramphenicol acetyltransferase gene driven by the murine alpha A-crystallin promoter in transgenic mice. Proc Natl Acad Sci USA. 1985;82:7815-7819.

3. Lee SW, Lee SH, Biswal S. Magnetic resonance reporter gene imaging. Theranostics. 2012;2:403-412. 
4. Blasberg RG, Gelovani J. Molecular-genetic imaging: a nuclear medicine-based perspective. Mol Imaging. 2002;1:280-300.

5. Luker GD, Luker KE. Optical imaging: current applications and future directions. J Nucl Med. 2008;49:1-4.

6. Chievitz O, Hevesy G. Radioactive indicators in the study of phosphorous metabolism in rats. Nature. 1935;136:754-755.

7. Hellebust A, Richards-Kortum R. Advances in molecular imaging: targeted optical contrast agents for cancer diagnostics. Nanomedicine (Lond). 2012;7:429-445.

8. Kircher MF, Gambhir SS, Grimm J. Noninvasive cell-tracking methods. Nat Rev Clin Oncol. 2011;8:677-688.

9. Collins SA, Hiraoka K, Inagaki A, Kasahara N, Tangney M. PET imaging for gene \& cell therapy. Curr Gene Ther. 2012;12:20-32.

10. Napp J, Mathejczyk JE, Alves F. Optical imaging in vivo with a focus on paediatric disease: technical progress, current preclinical and clinical applications and future perspectives. Pediatr Radiol. 2011;41:161-175.

11. Ntziachristos V. Going deeper than microscopy: the optical imaging frontier in biology. Nat Methods. 2010;7:603-614.

12. Troy T, Jekic-McMullen D, Sambucetti L, Rice B. Quantitative comparison of the sensitivity of detection of fluorescent and bioluminescent reporters in animal models. Mol Imaging. 2004;3:9-23.

13. Ray P, De A. Reporter gene imaging in therapy and diagnosis. Theranostics. 2012;2:333-334.

14. Gilad AA, Winnard PT Jr, van Zijl PC, Bulte JW. Developing MR reporter genes: promises and pitfalls. NMR Biomed. 2007;20:275-290.

15. Tjuvajev JG, Stockhammer G, Desai R, et al. Imaging the expression of transfected genes in vivo. Cancer Res. 1995;55:6126-6132.

16. Deroose CM, Chitneni SK, Gijsbers R, et al. Preliminary validation of varicella zoster virus thymidine kinase as a novel reporter gene for PET. Nucl Med Biol. 2012;39:1266-1274.

17. Ponomarev V, Doubrovin M, Shavrin A, et al. A human-derived reporter gene for noninvasive imaging in humans: mitochondrial thymidine kinase type $2 . \mathrm{J} \mathrm{Nucl}$ Med. 2007;48:819-826.

18. Campbell DO, Yaghoubi SS, Su Y, et al. Structure-guided engineering of human thymidine kinase 2 as a positron emission tomography reporter gene for enhanced phosphorylation of non-natural thymidine analog reporter probe. $J$ Biol Chem. 2012;287:446-454.

19. Likar Y, Zurita J, Dobrenkov K, et al. A new pyrimidine-specific reporter gene: a mutated human deoxycytidine kinase suitable for PET during treatment with acycloguanosine-based cytotoxic drugs. J Nucl Med. 2010;51:1395-1403.

20. Haberkorn U. Gene therapy with sodium/iodide symporter in hepatocarcinoma. Exp Clin Endocrinol Diabetes. 2001;109:60-62.

21. Altmann A, Kissel M, Zitzmann S, et al. Increased MIBG uptake after transfer of the human norepinephrine transporter gene in rat hepatoma. J Nucl Med. 2003;44:973-980.

22. Rogers BE, Zinn KR, Buchsbaum DJ. Gene transfer strategies for improving radiolabeled peptide imaging and therapy. Q J Nucl Med. 2000;44:208-223.

23. Olafsen T, Sirk SJ, Olma S, Shen CK, Wu AM. ImmunoPET using engineered antibody fragments: fluorine-18 labeled diabodies for same-day imaging. Tumour Biol. 2012;33:669-677

24. Liang Q, Satyamurthy N, Barrio JR, et al. Noninvasive, quantitative imaging in living animals of a mutant dopamine D2 receptor reporter gene in which ligand binding is uncoupled from signal transduction. Gene Ther. 2001;8:1490-1498.

25. Dai G, Levy O, Carrasco N. Cloning and characterization of the thyroid iodide transporter. Nature. 1996;379:458-460.

26. Pacholczyk T, Blakely RD, Amara SG. Expression cloning of a cocaine- and antidepressant-sensitive human noradrenaline transporter. Nature. 1991;350:350-354.

27. Moroz MA, Serganova I, Zanzonico P, et al. Imaging hNET reporter gene expression with ${ }^{124}$ I-MIBG. $J$ Nucl Med. 2007;48:827-836.

28. Buursma AR, Beerens AM, de Vries EF, et al. The human norepinephrine transporter in combination with ${ }^{11} \mathrm{C}$-m-hydroxyephedrine as a reporter gene/ reporter probe for PET of gene therapy. J Nucl Med. 2005;46:2068-2075.

29. Rogers BE, McLean SF, Kirkman RL, et al. In vivo localization of [ [ ${ }^{111}$ In]-DTPA-DPhe1-octreotide to human ovarian tumor xenografts induced to express the somatostatin receptor subtype 2 using an adenoviral vector. Clin Cancer Res. 1999;5:383-393.

30. Rogers BE, Parry JJ, Andrews R, Cordopatis P, Nock BA, Maina T. MicroPET imaging of gene transfer with a somatostatin receptor-based reporter gene and 94mTc-Demotate 1. J Nucl Med. 2005;46:1889-1897.
31. Bodei L, Ferone D, Grana CM, et al. Peptide receptor therapies in neuroendocrine tumors. J Endocrinol Invest. 2009;32:360-369.

32. Bushnell D. Treatment of metastatic carcinoid tumors with radiolabeled biologic molecules. J Natl Compr Canc Netw. 2009;7:760-764.

33. MacLaren DC, Gambhir SS, Satyamurthy N, et al. Repetitive, non-invasive imaging of the dopamine $\mathrm{D} 2$ receptor as a reporter gene in living animals. Gene Ther. 1999;6:785-791.

34. Guillermet-Guibert J, Lahlou H, Cordelier P, Bousquet C, Pyronnet S, Susini C. Physiology of somatostatin receptors. J Endocrinol Invest. 2005;28(11 suppl international):5-9.

35. Zhang H, Moroz MA, Serganova I, et al. Imaging expression of the human somatostatin receptor subtype-2 reporter gene with ${ }^{68}$ Ga-DOTATOC. $J$ Nucl Med. 2011;52:123-131.

36. Jacobs A, Voges J, Reszka R, et al. Positron-emission tomography of vectormediated gene expression in gene therapy for gliomas. Lancet. 2001;358:727729.

37. Dempsey MF, Wyper D, Owens J, et al. Assessment of ${ }^{123}$ I-FIAU imaging of herpes simplex viral gene expression in the treatment of glioma. Nucl Med Commun. 2006;27:611-617.

38. Peñuelas I, Mazzolini G, Boan JF, et al. Positron emission tomography imaging of adenoviral-mediated transgene expression in liver cancer patients. Gastroenterology. 2005;128:1787-1795

39. Yaghoubi SS, Jensen MC, Satyamurthy N, et al. Noninvasive detection of therapeutic cytolytic T cells with ${ }^{18} \mathrm{~F}$-FHBG PET in a patient with glioma. Nat Clin Pract Oncol. 2009;6:53-58.

40. Barton KN, Stricker H, Brown SL, et al. Phase I study of noninvasive imaging of adenovirus-mediated gene expression in the human prostate. Mol Ther. 2008;16: 1761-1769.

41. Yamamoto M, Curiel DT. Current issues and future directions of oncolytic adenoviruses. Mol Ther. 2010;18:243-250.

42. Yaghoubi SS, Campbell DO, Radu CG, Czernin J. Positron emission tomography reporter genes and reporter probes: gene and cell therapy applications. Theranostics. 2012;2:374-391.

43. Serganova I, Ponomarev V, Blasberg R. Human reporter genes: potential use in clinical studies. Nucl Med Biol. 2007;34:791-807.

44. Nguyen PK, Lan F, Wang Y, Wu JC. Imaging: guiding the clinical translation of cardiac stem cell therapy. Circ Res. 2011;109:962-979.

45. Ponomarev V, Doubrovin M, Lyddane C, et al. Imaging TCR-dependent NFATmediated T-cell activation with positron emission tomography in vivo. Neoplasia. 2001;3:480-488.

46. Lyons SK, Meuwissen R, Krimpenfort P, Berns A. The generation of a conditional reporter that enables bioluminescence imaging of Cre/loxP-dependent tumorigenesis in mice. Cancer Res. 2003;63:7042-7046.

47. Uhrbom L, Nerio E, Holland EC. Dissecting tumor maintenance requirements using bioluminescence imaging of cell proliferation in a mouse glioma model. Nat Med. 2004;10:1257-1260.

48. Lyons SK, Lim E, Clermont AO, et al. Noninvasive bioluminescence imaging of normal and spontaneously transformed prostate tissue in mice. Cancer Res. 2006;66:4701-4707.

49. Wang Y, Iyer M, Annala A, Wu L, Carey M, Gambhir SS. Noninvasive indirect imaging of vascular endothelial growth factor gene expression using bioluminescence imaging in living transgenic mice. Physiol Genomics. 2006;24:173-180.

50. Doubrovin M, Ponomarev V, Beresten $\mathrm{T}$, et al. Imaging transcriptional regulation of p53-dependent genes with positron emission tomography in vivo. Proc Natl Acad Sci U S A. 2001;98:9300-9305.

51. Mayer-Kuckuk P, Banerjee D, Malhotra S, et al. Cells exposed to antifolates show increased cellular levels of proteins fused to dihydrofolate reductase: a method to modulate gene expression. Proc Natl Acad Sci U S A. 2002;99: 3400-3405.

52. Luker GD, Sharma V, Pica CM, Prior JL, Li W, Piwnica-Worms D. Molecular imaging of protein-protein interactions: controlled expression of $\mathrm{p} 53$ and large Tantigen fusion proteins in vivo. Cancer Res. 2003;63:1780-1788.

53. Doubrovin M, Che JT, Serganova I, et al. Monitoring the induction of heat shock factor 1 /heat shock protein 70 expression following 17-allylaminodemethoxygeldanamycin treatment by positron emission tomography and optical reporter gene imaging. Mol Imaging. 2012;11:67-76. 